

\title{
Engineering analysis error estimation when removing finite-sized features in nonlinear elliptic problems
}

\author{
Ming $\mathrm{Li}^{*}$, Shuming Gao \\ State Key Laboratory of CADECG, Zhejiang University, China \\ Ralph R. Martin \\ School of Computer Science 6 Informatics, Cardiff University, UK
}

\begin{abstract}
The paper provides novel approaches for a posteriori estimation of goal-oriented engineering analysis error caused by removing finite-sized negative features from a complex model, in the case of analysis of nonlinear elliptic physical phenomena. The features may lie within the model's interior or along its boundary, and may be constrained with either Neumann or Dirichlet boundary conditions. The main use is for deciding whether detail design features can be removed from a model, to simplify meshing and engineering analysis, without unduly affecting analysis results.

Error estimates are found using adjoint theory. Using a rigorous mathematical derivation, the error is first reformulated as a local quantity defined over the boundary of the feature to be suppressed, via linearization and Green's theorem. This intermediate result still involves unknown terms, which we overcome in three ways. In one, an approximate upper bound of the error is obtained rigorously utilizing classical theories of differential operators; the others are heuristic practical approaches. Performance and effectivity of these three different approaches is examined on 2D and 3D internal and boundary features, with Neumann and Dirichlet boundary conditions.
\end{abstract}

Key words: modification error, defeaturing, analysis-dependent simplification, semilinear elliptic equation, CAD/CAE integration

\section{Introduction}

Engineering design and analysis are interlinked. Physical performance of an engineering design is verified via engineering analysis, and results of engineering analysis in turn drive design modifications. Achieving integration between engineering design and analysis is a challenging task, and is estimated to occupy $80 \%$ of the whole engineering analysis process. The core reasons behind this difficulty lie in both model complexity and the different geometric representations used for design and analysis. Computer aided design (CAD) models typically use boundary representation (B-rep) giving a watertight volume bounded by a set of parametric surfaces. Engineering analysis is usually performed on discrete volumetric meshes, allowing numeric approximation to a boundary value problem (a set of PDEs constrained by certain boundary conditions) via finite element analysis (FEA), finite differences, etc. Much effort has been devoted to converting CAD models into mesh mod-

\footnotetext{
* Corresponding author: liming@cad.zju.edu.cn
}

els [20]. Recently, Hughes [14] proposed the novel concept of isogeometric analysis (IGA) allowing unification of design and analysis representations, via NURBS or T-splines, overcoming the need for conversion.

Integrating design and analysis for CAD/CAE (Computer Aided Engineering) typically involves more than mesh generation. Engineering analysis is often performed on idealized or simplified geometries, as (i) meshing is easier and less likely to fail, (ii) the meshes are simpler, needing fewer small elements to capture features, and (iii) as a consequence, analysis is quicker; it may also be more robust due to higher mesh quality. Consider the engine and the simplified version after certain detailed features have been removed in Fig. 1. The simplified mesh has less than 1/4 of the number of vertices, which could potentially reduce downstream analysis time by a factor of $(1 / 4)^{3}=1 / 64$.

Due to geometric complexity, and variability of physical phenomena in sensitivity to geometric detail, the task of analysis-dependent geometry simplification is a timeconsuming task; it is usually performed manually in industrial practice. It is estimated to account for $57 \%$ of overall 


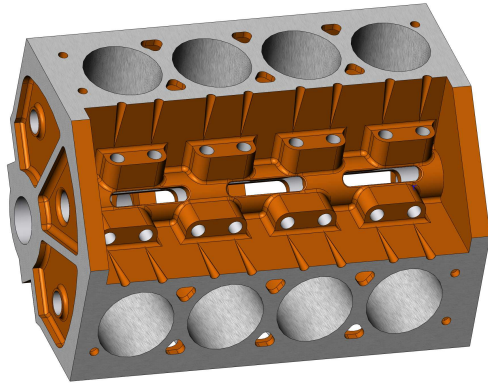

(a) Original model

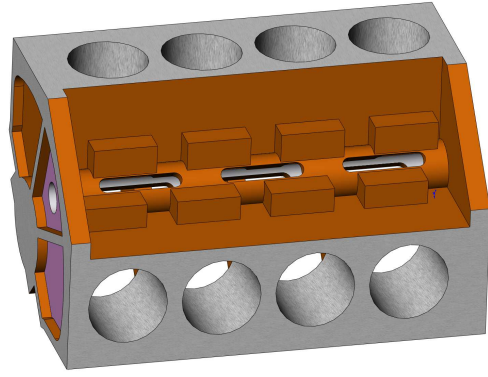

(b) Simplified model

Fig. 1. An engine block and a simplified model.

analysis time, while mesh generation accounts for $23 \%$; it is still not a fully solved problem $[4,22,24,26]$.

In academia, geometry simplification for analysis has been studied as a topic in both geometric modeling, and engineering analysis. The geometric modeling point of view focuses on geometric operations or algorithms to carry out simplification, but usually ignores effects on analysis accuracy. Criteria for guiding simplification are rooted in the model's geometry, and ignore physical properties and the physical problem being solved. Such approaches cannot ensure analysis will produce results of a desired accuracy.

Engineering analysis mainly focuses on the existence, uniqueness, and accuracy of numerical solutions through a priori or a posteriori error estimates. Generally, two types of errors have been studied. Numerical approximation errors are incurred by computing discrete solutions for boundary value problems [16]. Modeling errors are caused by using simplified mathematical equations to model physical phenomena [19]. Both approaches, however, assume that the underlying geometry upon which analysis is performed is exact, while geometric simplification involves changes to the underlying geometry.

The core issue in analysis-dependent geometry simplification is to quantitatively estimate the engineering analysis error caused by defeaturing, or the modification error for short. Solving an engineering analysis problem by utilizing solutions of the defeatured model was first considered in 1994 by Keller [15]. More recently, the work of Suresh et al $[12,11,27]$ has made further progress on this topic, as has work by Ferrandes et al [9] and Li et al [17]. However, these previous studies are mainly limited to linear problems, e.g. Poisson's equation and linear elasticity, and are also limited to features with (homogeneous) Neumann boundary conditions (BCs).

Modification error estimation for nonlinear elliptic problems was first studied in [18], which however only considered Neumann BCs, using the dual weighted residual (DWR) method. In this paper, we derive error estimates based on adjoint theory, which can handle features with either Neumann BCs or Dirichlet BCs. Dirichlet BCs have been little studied other than in [12], which only considers linear Poisson equations with a special outer BC (solution equal to zero). Here we build upon their idea in converting goaloriented modification error into a local quantity defined over the boundary of the suppressed feature. This idea was originally proposed for the Poisson equation; we extend it to the nonlinear elliptic case - see also Section 3.1. After this reformulation, we develop novel approaches for features within the model's interior or along its boundary. Our approach covers a wide range of situations, while the approach in [12] for Neumann BC is essentially only applicable to a single internal feature, due to the use of exterior approximation, which is undefined for multiple features or features lying on the boundary of an object.

Other related work on shape sensitivity analysis (SSA) [7] and topological sensitivity analysis (TSA) [25,2] mainly focuses on infinitesimal geometry changes, while we consider features of finite size. However, in this context, a rule-based principle was also proposed by Russ et al [23] to simplify CAD models to facilitate engineering analysis.

This paper proposes novel approaches for modification error estimation. Our methods cover a wide range of practical industrial applications, many of which involve removing finite-sized negative features within a model's interior or on its boundary, with either Neumann or Dirichlet BCs, for nonlinear elliptic problems. The paper specifically focuses on semilinear elliptic equations, which represent a wide range of physical phenomena, such as stationary heat conduction, wave propagation, and others [3]. Using a rigorous mathematical derivation, the error is first expressed as a local quantity defined over the boundary of the feature to be suppressed, by exploiting adjoint theory and a linearization approximation process. An approximate upper bound for the modification error is obtained, and two other heuristic numerical approaches are also presented for estimating the error, which are of use in practical computations. These three different approaches are experimentally validated for $2 \mathrm{D}$ and $3 \mathrm{D}$ internal and boundary features, with either Neumann or Dirichlet BCs.

The problem of estimating modification error is stated in Section 2. Concrete approaches for error estimation are detailed in Section 3. Numerical experiments are presented in Section 4, and conclusions are drawn in Section 5.

\section{The problem of estimating modification error}

Following [27,18], the problem of a posteriori goaloriented modification error estimation is now described.

Unlike previous studies that focus on linear cases, we con- 
sider more challenging nonlinear elliptic engineering analysis problems. We restrict our attention to a semilinear elliptic boundary value problem [3]. Examples that fall into this important mathematical category can be found in a variety of contexts in geometry, physics, mechanics, engineering and, more recently, life sciences, representing, for example, stationary heat conduction or wave conduction. Nonlinear phenomena must be taken into account to understand these fields [3]. Extension of the basic framework to other nonlinear elliptic problems is discussed in Section 5.

The nonlinear problem is defined over an original complex geometry, whose corresponding field solutions are assumed to be time consuming or hard to compute, or even intractable. By removing a set of features that form part of the original geometry, a simplified geometry is obtained, whose corresponding field solutions are presumed to be much easier to determine. Examples of an original complex geometry, and a corresponding simplified geometry, are shown in Fig. 1.

In this paper, we only study elimination of negative features (i.e. where material has been removed), which may be contained within the geometry's interior or lie on its outer boundary. When only negative features exist, the original geometry is entirely contained within the simplified geometry, a useful property which no longer holds if positive features are also present. Negative features are dominant in engineering components: many engineering components are created by removing materials from a blank such as a block (again see Fig. 1). A heuristic approach to extending the approaches here to positive features could potentially follow the ideas in [17]. The class of negative features considered in this paper is quite general - they can be of finitesize, within the geometry's interior or on its boundary, and subject to either Neumann or Dirichlet BCs.

The problem is now described for a single internal feature $\omega$ contained within the interior of the original geome$\operatorname{try} \Omega-\omega$ in $n$-dimensional Euclidean space $\mathbb{R}^{n}, n=2$ or 3 . See Fig. 2(a). Extension to boundary features or multiple features is straightforward, and will be explained in Section 3.4. Removing $\omega$ from $\Omega-\omega$ leaves a simplified geometry $\Omega$ as shown in Fig. 2(b). The boundary of $\Omega$ or $\omega$ is denoted by $\partial \Omega$ or $\partial \omega$ respectively. As $\omega$ is fully contained within $\Omega$, we have $\partial(\Omega-\omega)=\partial \Omega+\partial \omega$. We assume that both $\Omega-\omega$ and $\Omega$ are bounded connected open regions with Lipschitz boundaries [8], to ensure the validity of application of various classical results used in Section 3.

Over the original complex geometry $\Omega-\omega$, a nonlinear second order elliptic equation with solution $u$ is defined: find the field solution $u$ such that

$$
\begin{cases}L u=f & \text { in } \Omega-\omega, \\ B u=b & \text { on } \partial \Omega, \\ D u=d \text { or } u_{d} & \text { on } \partial \omega,\end{cases}
$$

where $L$ is an abstract nonlinear differential operator governing the second order elliptic phenomenon, where we specifically take

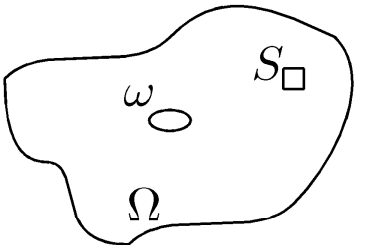

(a) Original geometry $\Omega-\omega$

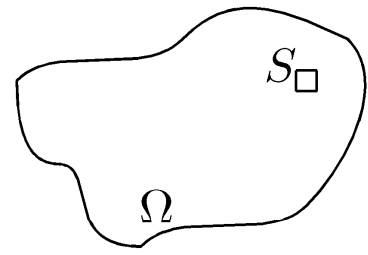

(b) Simplified geometry $\Omega$
Fig. 2. Engineering analysis problems for original and simplified geometries.

$$
L u=-\operatorname{div}(\nabla u)+u^{3} .
$$

Boundary operators $B$ and $D$ determine either Neumann or Dirichlet BCs on the outer and inner boundaries $\partial \Omega$ and $\partial \omega$. The latter may be subject to either (a) Neumann or (b) Dirichlet BCs (if mixed, they can be considered separately): $D u=d$ or $u_{d}$ stands for

$$
\text { (a) } \partial u / \partial n=d, \quad \text { or } \quad(b) u=u_{d} \quad \text { on } \partial \omega .
$$

The problem and its BCs are characterized by the continuous functions $f, b, d$ and $u_{d}$.

For the above problem, uniqueness and stability of the solution can be readily guranteed [6]. Thus, we can straightforwardly focus on the error estimate.

Removing $\omega$ from $\Omega-\omega$ leaves a simplified geometry with different solution $u_{0}$ satisfying

$$
\begin{cases}L u_{0}=f & \text { in } \Omega, \\ B u_{0}=b & \text { on } \partial \Omega .\end{cases}
$$

The associated BCs $D u=d$ over $\partial \omega$ have disappeared due to the removal of the internal feature $\omega$.

The geometric difference between the original geometry $\Omega-\omega$ and its simplification $\Omega$, essentially the difference of the underlying computational domains, perturbs the engineering analysis problem defined over them. As in traditional approaches to measuring approximation errors or modeling errors, this solution difference, or modification error for short, is described in terms of goal-oriented error which records changes in quantities of particular engineering or scientific interest. Compared to a global error measure in an energy norm, goal-oriented error is much more useful in understanding effects on local values of interest, such as pointwise temperature or maximal stress over a certain portion of the shape's boundary.

Specifically, let us assume we are interested in a particular local quantity of interest prescribed over a local region $S \in \Omega-\omega$. In general form this may be written as follows:

$$
Q(u)=\int_{S} q(u) d S,
$$

where $q(\cdot)$ may be a linear or nonlinear function defined over $S$. We wish to estimate the difference in this local quantity caused by defeaturing, i.e.

$$
\delta Q=e(\Omega-\omega, \Omega)=Q(u)-Q\left(u_{0}\right) .
$$

It is permissible to use the solution $u_{0}$ but we do not want to explicitly use the solution $u$ for the original geometry, as this would defeat the purpose of defeaturing. 


\section{Error estimation}

Our approach to estimating the modification error is based on adjoint theory. The target quantity $Q(u)-Q\left(u_{0}\right)$ is reformulated as a local quantity defined over the boundary of the suppressed feature, by extending results for the Poisson equation in [12] to the nonlinear elliptic case. The result, stated in Theorem 1, is the basis of the desired error estimate but still involves unknown terms. Novel approximate bounds for these unknown terms are then derived for features with Neumann or Dirichlet BCs, and presented in Theorems 2 and 3. Following Theorem 1, we also propose two other novel heuristic approaches for providing modification error estimates in Section 3.4.

\subsection{Error expressions based on adjoint theory}

We first consider how to reformulate the target quantity $Q(u)-Q\left(u_{0}\right)$ as a local quantity defined over the boundary of the suppressed feature. The main steps are linearization and use of Green's theorem with integration by parts. Specifically, we first build linear (approximate) governing equations of the solution error $u-u_{0}$, and their adjoints, in Lemma 1. The linearization process, in combination with a linear approximation to $Q(u)-Q\left(u_{0}\right)$, allows us to reformulate the target modification error as a local quantity defined over the boundary of the suppressed feature using adjoint theory and Green's theorem, following a similar procedure to that used in [12].

We now briefly introduce adjoint theory. An adjoint solution to an adjoint problem is defined with respect to an originally defined prime problem. See, for example, Eq. (1) or (3). Adjoint solutions are typically used as weight factors in a posteriori goal-oriented error estimates in traditional FE approximation [5] or modeling approximation [19]. Here, local residuals of the computed solutions are weighted by the adjoint solutions, which measure the dependence of the error on the local residual.

If the target functional $Q(\cdot)$ is linear, the adjoint formulation for a linear differential operator $L$ in Eq. (1) may be described in a similar form to that for the prime problem by replacing $f$ (defined in Eq. (1)) by $q$ (defined in Eq. (5)). Deriving such an adjoint operator for a nonlinear differential operator $L$ or target quantity $Q(\cdot)$ is not a trivial task, and is generally obtained using the primal-dual equivalence condition, as systematically studied by Giles and Suli [10].

For the specific nonlinear second order elliptic equation problem in Eq. (1), the corresponding adjoint problem, with respect to a linear or nonlinear target function $Q(\cdot)$, has the following form $[6,13]$ : find the solution $p$ such that

$$
\begin{cases}-\operatorname{div}(\nabla p)+3 u^{2} p=0 & \text { in } \Omega-\omega-S, \\ -\operatorname{div}(\nabla p)+3 u^{2} p=q^{\prime}(u) & \text { in } S \\ B p=0 & \text { on } \partial \Omega \\ D p=0 & \text { on } \partial \omega .\end{cases}
$$

Note that in the above adjoint formulation, the prime solution $u$ is also involved in the first and second equations, and values of the BCs over the internal and outer boundaries become zero. This occurrence of $u$ only arises in nonlinear cases, and makes the corresponding error estimation problem much more challenging.

Similarly, the corresponding adjoint formulation for Eq. (3) is: find the solution $p_{0}$ such that,

$$
\begin{cases}-\operatorname{div}\left(\nabla p_{0}\right)+3 u_{0}^{2} p_{0}=0 & \text { in } \Omega-S, \\ -\operatorname{div}\left(\nabla p_{0}\right)+3 u_{0}^{2} p_{0}=q^{\prime}\left(u_{0}\right) & \text { in } S, \\ B p_{0}=0 & \text { on } \partial \Omega .\end{cases}
$$

In order to properly estimate the modification error in Eq. (5), linear approximations to the governing equation of both the prime solution error $e_{0}=u-u_{0}$, and the adjoint error $\epsilon_{0}=p-p_{0}$ are first built, which, together with a linearization of the local target quantity $Q(u)$, linearize the problem, as we now explain.

Firstly, approximate linear governing equations for $e_{0}, \epsilon_{0}$ are derived below.

Lemma 1 The solution error $e_{0}=u-u_{0}$ and adjoint error $\epsilon_{0}=p-p_{0}$ are approximately governed by the following linear equations with prescribed BCs,

$$
\begin{cases}-\operatorname{div}\left(\nabla e_{0}\right)+3 u_{0}^{2} e_{0}=0 & \text { in } \Omega-\omega, \\ B e_{0}=0 & \text { on } \partial \Omega \\ D e_{0}=d \text { or } u_{d}-D u_{0} & \text { on } \partial \omega .\end{cases}
$$

and

$$
\begin{cases}-\operatorname{div}\left(\nabla \epsilon_{0}\right)+3 u_{0}^{2} \epsilon_{0}=0 & \text { in } \Omega-\omega, \\ B \epsilon_{0}=0 & \text { on } \partial \Omega, \\ D \epsilon_{0}=-D p_{0} & \text { on } \partial \omega .\end{cases}
$$

Proof: We only prove Eq. (8); Eq. (9) can be proved similarly. The validity of the BCs over the outer and internal boundaries follows directly. We now prove the first equation in Eq. (8) over the interior $\Omega-\omega$. From Eqs. (1) and (3),

$$
L u=-\operatorname{div}(\nabla u)+u^{3}=f, \quad L u_{0}=-\operatorname{div}\left(\nabla u_{0}\right)+u_{0}^{3}=f .
$$

Subtracting the above two equations, we have

$$
L u-L u_{0}=-\operatorname{div}\left(\nabla\left(u-u_{0}\right)\right)+\left(u^{3}-u_{0}^{3}\right)=0 .
$$

The Taylor expansion of $u^{3}-u_{0}^{3}$ when $u-u_{0}$ is small is

$$
u^{3}-u_{0}^{3}=3 u_{0}^{2}\left(u-u_{0}\right)+O\left(u-u_{0}\right) .
$$

Discarding the higher order term gives

$$
L u-L u_{0}=-\operatorname{div}\left(\nabla\left(u-u_{0}\right)\right)+3 u_{0}^{2}\left(u-u_{0}\right)=0 .
$$

Hence, as required,

$$
-\operatorname{div}\left(\nabla e_{0}\right)+3 u_{0}^{2} e_{0}=0
$$

From the above lemma, the modification error $\delta Q=$ $Q(u)-Q\left(u_{0}\right)$ can be approximated via a linearization process, using adjoint theory. Specifically, after finding a linear 
approximation to $Q(u)-Q\left(u_{0}\right)$, the adjoint solution can then be introduced into the error expression. Then, following a similar procedure to one in [12], performing integration by parts twice, and utilizing the relation between solutions $u$ and $u_{0}$ (they are governed by the same differential operator within the model interior and constrained by the same BCs except on the internal feature boundary), the modification error can be ultimately expressed as a local quantity defined over the internal feature boundary. This is stated in the following theorem, followed by its proof.

Theorem 1 The modification error $\delta Q$ in Eq. (5) for an internal feature $\omega$ can be estimated as a local quantity defined over the feature boundary $\partial \omega$ using adjoint solutions when $u-u_{0}$ is small:

$\delta Q \approx-\int_{\partial \omega} \nabla p_{0} \cdot n\left(u-u_{0}\right) d S+\int_{\partial \omega} p_{0} \nabla\left(u-u_{0}\right) \cdot n d S$.

Proof: Denote $\bar{\Omega}=\Omega-\omega$ for simplicity. Using Taylor expansion, a linear approximation to the target quantity $\delta Q$ can be obtained when $u-u_{0}$ is small:

$$
\begin{aligned}
\delta Q & =\int_{S}\left(q(u)-q\left(u_{0}\right)\right) d V \\
& =\int_{S}\left(q^{\prime}\left(u_{0}\right)\left(u-u_{0}\right)+O\left(u-u_{0}\right)\right) d V .
\end{aligned}
$$

Discarding the higher order term $O\left(u-u_{0}\right)$, and taking into account Eq. (7), we have

$$
\begin{aligned}
\delta Q & \approx-\int_{S}\left(\operatorname{div}\left(\nabla p_{0}\right)-3 u_{0}^{2} p_{0}\right)\left(u-u_{0}\right) d V \\
& -\int_{\bar{\Omega}-S}\left(\operatorname{div}\left(\nabla p_{0}\right)-3 u_{0}^{2} p_{0}\right)\left(u-u_{0}\right) d V .
\end{aligned}
$$

After performing two steps of integration by parts, we get

$$
\begin{aligned}
\delta Q \approx & -\int_{\partial \bar{\Omega}} \nabla p_{0} \cdot n\left(u-u_{0}\right) d S+\int_{\bar{\Omega}} \nabla p_{0} \cdot \nabla\left(u-u_{0}\right) d V \\
& \left.+\int_{\bar{\Omega}} 3 u_{0}^{2} p_{0}\left(u-u_{0}\right)\right) d V \\
= & -\int_{\partial \bar{\Omega}} \nabla p_{0} \cdot n\left(u-u_{0}\right) d S+\int_{\partial \bar{\Omega}} p_{0} \nabla\left(u-u_{0}\right) \cdot n d S \\
& -\int_{\bar{\Omega}}\left(\operatorname{div}\left(\nabla\left(u-u_{0}\right)\right) p_{0}-3 u_{0}^{2} p_{0}\left(u-u_{0}\right)\right) d V .
\end{aligned}
$$

From Eq. (8), the third term in the above equation vanishes, so

$\delta Q \approx-\int_{\partial \bar{\Omega}} \nabla p_{0} \cdot n\left(u-u_{0}\right) d S+\int_{\partial \bar{\Omega}} p_{0} \nabla\left(u-u_{0}\right) \cdot n d S$.

Furthermore, given Neumann BC over $\partial \Omega$,

$$
\nabla p_{0} \cdot n=\nabla\left(u-u_{0}\right) \cdot n=0 \quad \text { over } \quad \partial \Omega,
$$

or for Dirichlet BC over $\partial \Omega$,

$$
p_{0}=u-u_{0}=0, \quad \text { over } \partial \Omega,
$$

considering the part of Eq. (11) along $\partial \Omega$ gives

$$
-\int_{\partial \Omega} \nabla p_{0} \cdot n\left(u-u_{0}\right) d S+\int_{\partial \Omega} p_{0} \nabla\left(u-u_{0}\right) \cdot n d S=0 .
$$

Consequently, as $\partial \bar{\Omega}=\partial(\Omega-\omega)=\partial \Omega+\partial \omega$, Eq. (11) gives, as required

$\delta Q \approx-\int_{\partial \omega} \nabla p_{0} \cdot n\left(u-u_{0}\right) d S+\int_{\partial \omega} p_{0} \nabla\left(u-u_{0}\right) \cdot n d S$.

A similar procedure shows that Theorem 1 also holds true for the case of a boundary feature. Later results in this paper based on Theorem 1 do not explicitly assume internal features, and thus also hold true for boundary features. We particularly note that the approximate equality in Eq. (10) only arises due to linearization of $Q(u)-Q\left(u_{0}\right)$ in Eq. (11), and of the governing equations for $e_{0}$ and $\epsilon_{0}$. The equality in Eq. (10) holds exactly if $L$ is linear and if $Q(u)$ is linear.

Theorem 1 reformulates the modification error in terms of a local quantity defined over the feature boundary $\partial \omega$ using adjoint solutions. This estimate cannot yet be computed directly since it still involves the unknown full solution $u$. Consider Eq. (10). For Neumann BC on $\partial \omega$, the value of $\nabla\left(u-u_{0}\right) \cdot n$ in the second term is known, and the value of $u-u_{0}$ in the first term is unknown. On the other hand, for Dirichlet BC on $\partial \omega$, the value of $u-u_{0}$ in the first term is known, and value of $\left(u-u_{0}\right) \cdot n$ in the second term is unknown. In either case, one term in Eq. (10) is unknown and needs to be further estimated, which we now consider.

Before we start, three norms are introduced. These are the energy norm:

$\|v\|_{E}=\sqrt{\alpha(v, v)}$, for $\alpha(u, v)=\int_{\Omega-\omega}\left(\nabla u \cdot \nabla v+3 u_{0}^{2} u v\right) d V$,

the $L^{p}$-norm:

$$
\|v\|_{L^{p}(\Omega)}=\left(\int_{\partial \omega}|v|^{p} d S\right)^{1 / p}
$$

and the $H^{k}$-norm:

$$
\|v\|_{H^{k}(\Omega)}=\left(\sum_{|\kappa| \leq k}\left\|D^{\kappa} v\right\|_{L^{2}(\Omega)}^{2}\right)^{1 / 2}
$$

where $\kappa$ is a multi-index, and

$$
D^{\kappa} v=\frac{\partial^{|\kappa|} v}{\partial x_{1}^{\kappa_{1}} \ldots \partial x_{n}^{\kappa_{n}}}
$$

\subsection{Error estimation for features with Neumann BCs}

In this section, we give a novel approach to further estimate the quantities in Eq. (10) when the internal feature $\omega$ has Neumann BCs, $\nabla u \cdot n=d$ over $\partial \omega$. In this case, the second term on the right hand side of Eq. (10) is known:

$$
\int_{\partial \omega} p_{0} \nabla\left(u-u_{0}\right) \cdot n d S=\int_{\partial \omega}\left(d-d_{0}\right) p_{0} d S
$$

for $d_{0}=\nabla u_{0} \cdot n$. We only need to estimate the first, unknown, term, as below.

Theorem 2 When feature $\omega$ has Neumann BC, specifically $\nabla u \cdot n=d$ on $\partial \omega$, the modification error in Eq. (5) is approximately bounded below by 
$|\delta Q| \leq\left|\int_{\partial \omega}\left(d-d_{0}\right) p_{0} d S\right|+C_{N}^{2}\left\|\nabla p_{0} \cdot n\right\|_{L^{2}(\partial \omega)}\left\|d-d_{0}\right\|_{L^{2}(\partial \omega)}$,

where $d_{0}=\nabla u_{0} \cdot n$, and $C_{N}$ is a constant only dependent on the geometry of $\Omega-\omega$, whose practical computation will be discussed in Section 3.4.

Proof: We first estimate the energy norm of the solution error $e_{0}=u-u_{0}$, and then use it to estimate $\delta Q$ based on Eq. (10).

Multiplying the first line of Eq. (9) by a functional $v$ and performing integration by parts gives the following weak form for $e_{0}$ : find solution $e_{0} \in V$ such that

$$
\int_{\Omega-\omega}\left(\nabla e_{0} \cdot \nabla v+3 u_{0}^{2} e_{0} v\right) d V=\int_{\partial \omega}\left(d-d_{0}\right) v d s, v \in V,
$$

where $V$ is the appropriate Sobolev space [8].

From the trace theorem (see [8]) and the equivalence of the $H^{1}$ norm and the energy norm, a constant $C_{N}$ exists such that

$$
\|v\|_{L^{2}(\partial \omega)} \leq C_{N}\|v\|_{E}, \quad v \in V
$$

Thus,

$$
\begin{aligned}
\left|\alpha\left(e_{0}, v\right)\right| & =\left|\int_{\partial \omega}\left(d-d_{0}\right) v d s\right| \\
& \leq\left\|d-d_{0}\right\|_{L^{2}(\partial \omega)}\|v\|_{L^{2}(\partial \omega)} \\
& \leq C_{N}\left\|d-d_{0}\right\|_{L^{2}(\partial \omega)}\|v\|_{E} .
\end{aligned}
$$

Replacing $v$ by $e_{0}$ in the above equation gives

$$
\left\|e_{0}\right\|_{E}^{2}=\alpha\left(e_{0}, e_{0}\right) \leq C_{N}\left\|d-d_{0}\right\|_{L^{2}(\partial \omega)}\left\|e_{0}\right\|_{E},
$$

or equivalently,

$$
\left\|e_{0}\right\|_{E} \leq C_{N}\left\|d-d_{0}\right\|_{L^{2}(\partial \omega)}
$$

Thus, as $e_{0}=u-u_{0}$, the first unknown term in Eq. (10) may be estimated to be no more than the quantity below:

$$
\begin{aligned}
\mid \int_{\partial \omega} & \nabla p_{0} \cdot n e_{0} d S \mid \leq\left\|\nabla p_{0} \cdot n\right\|_{L^{2}(\partial \omega)}\left\|e_{0}\right\|_{L^{2}(\partial \omega)} \\
& \leq\left\|\nabla p_{0} \cdot n\right\|_{L^{2}(\partial \omega)} C_{N}\left\|e_{0}\right\|_{E} \\
& \leq C_{N}^{2}\left\|\nabla p_{0} \cdot n\right\|_{L^{2}(\partial \omega)}\left\|d-d_{0}\right\|_{L^{2}(\partial \omega)}
\end{aligned}
$$

By further taking into account Eqs. (13) and (10), the triangle inequality gives Theorem 2 .

\subsection{Error estimation for features with Dirichlet BCs}

We next give a novel approach to further estimate the quantities in Eq. (10) when the internal feature $\omega$ has Dirichlet BCs, $u=u_{d}$ over $\partial \omega$. In this case, the first term on the right side of Eq. (10) is known, so

$$
-\int_{\partial \omega} \nabla p_{0} \cdot n\left(u-u_{0}\right) d S=-\int_{\partial \omega} \nabla p_{0} \cdot n\left(u_{d}-u_{0}\right) d S,
$$

and we only need to further estimate the unknown, second, term. As a result, estimation of the modification error in this case is given below.
Theorem 3 When feature $\omega$ has Dirichlet BC, specifically $u=u_{d}$ on $\partial \omega$, the modification error in Eq. (5) is approximately bounded below by:

$$
\begin{aligned}
|\delta Q| & \leq\left|\int_{\partial \omega} \nabla p_{0} \cdot n\left(u_{d}-u_{0}\right) d S\right| \\
& +C_{D}\left\|p_{0}\right\|_{L^{2}(\partial \omega)}\left(\left\|u_{d}-u_{0}\right\|_{L^{2}(\partial \omega)}+\nu(\tilde{e})\right),
\end{aligned}
$$

where $\nu(\tilde{e})=\|\tilde{e}\|_{L^{2}(\Omega-\omega)}+C_{0}\left\|\operatorname{div}(\nabla \tilde{e})-3 u_{0}^{2} \tilde{e}\right\|_{L^{2}(\Omega-\omega)}$, $\tilde{e}$ is any function satisfying $E q .(19)$, and $C_{0}, C_{D}$ are constants only dependent on the geometry of $\Omega-\omega$, whose practical computation will be discussed in Section 3.4.

Proof: This follows the proof for Neumann BC, but is more challenging. A strategy of reformulating the Dirichlet term with respect to $e_{0}$ over the internal feature boundary $\partial \omega$ using the source term of its governing equation is applied. We first consider estimation of $\left\|e_{0}\right\|_{L^{2}(\Omega-\omega)}$. Choose a function $\tilde{e}$ satisfying the following BCs:

$$
B \tilde{e}=0 \quad \text { on } \partial \Omega, \quad \tilde{e}=u_{d}-u_{0} \quad \text { on } \partial \omega .
$$

Setting $\bar{e}=e_{0}-\tilde{e}$, Eq. (8) gives the following equation for $\bar{e}$ :

$$
\begin{cases}-\operatorname{div}(\nabla \bar{e})+3 u_{0}^{2} \bar{e}=\operatorname{div}(\nabla \tilde{e})-3 u_{0}^{2} \tilde{e} & \text { in } \Omega-\omega, \\ B \bar{e}=0 & \text { on } \partial \Omega, \\ \bar{e}=0 & \text { on } \partial \omega .\end{cases}
$$

$H^{2}$-regularity of elliptic operators (see [8]) gives

$\|\bar{e}\|_{L^{2}(\Omega-\omega)} \leq\|\bar{e}\|_{H^{2}(\Omega-\omega)} \leq C_{0}\left\|\operatorname{div}(\nabla \tilde{e})-3 u_{0}^{2} \tilde{e}\right\|_{L^{2}(\Omega-\omega)}$, which implies

$$
\left\|e_{0}\right\|_{L^{2}(\Omega-\omega)} \leq\|\bar{e}\|_{L^{2}(\Omega-\omega)}+\|\tilde{e}\|_{L^{2}(\Omega-\omega)} \leq \nu(\tilde{e})
$$

for $\nu(\tilde{e})=\|\tilde{e}\|_{L^{2}(\Omega-\omega)}+C_{0}\left\|\operatorname{div}(\nabla \tilde{e})-3 u_{0}^{2} \tilde{e}\right\|_{L^{2}(\Omega-\omega)}$.

Next we estimate $\left\|\nabla e_{0}\right\|_{L^{2}(\partial \omega)}$. From the trace theorem, a constant $C_{1}$ can be found such that

$$
\|\nabla v\|_{L^{2}(\partial \omega)} \leq C_{1}\|v\|_{H^{1}(\Omega-\omega)}, \quad v \in V .
$$

$H^{2}$-regularity and the form of the governing equation of $e_{0}$ in Eq. (8) show that a constant $C_{2}$ can be found such that

$$
\left\|e_{0}\right\|_{H^{2}(\Omega-\omega)} \leq C_{2}\left(\left\|u_{d}-u_{0}\right\|_{L^{2}(\partial \omega)}+\left\|e_{0}\right\|_{L^{2}(\Omega-\omega)}\right) .
$$

Combining Eqs. (20), (21) and (22) gives

$$
\begin{aligned}
\left\|\nabla e_{0}\right\|_{L^{2}(\partial \omega)} & \leq C_{1}\left\|e_{0}\right\|_{H^{1}(\Omega-\omega)} \leq C_{1}\left\|e_{0}\right\|_{H^{2}(\Omega-\omega)} \\
& \leq C_{D}\left(\left\|u_{d}-u_{0}\right\|_{L^{2}(\partial \omega)}+\nu(\tilde{e})\right),
\end{aligned}
$$

where $C_{D}=C_{1} C_{2}$.

Ultimately, we have

$$
\begin{aligned}
& \left|\int_{\partial \omega} p_{0} \nabla\left(u-u_{0}\right) \cdot n d S\right|=\left|\int_{\partial \omega} p_{0} \nabla e_{0} \cdot n d S\right| \\
& \leq\left\|p_{0}\right\|_{L^{2}(\partial \omega)}\left\|\nabla e_{0} \cdot n\right\|_{L^{2}(\partial \omega)} \\
& \leq\left\|p_{0}\right\|_{L^{2}(\partial \omega)}\left\|\nabla e_{0}\right\|_{L^{2}(\partial \omega)} \\
& \leq C_{D}\left\|p_{0}\right\|_{L^{2}(\partial \omega)}\left(\left\|u_{d}-u_{0}\right\|_{L^{2}(\partial \omega)}+\nu(\tilde{e})\right) .
\end{aligned}
$$

By further taking into account Eqs. (17) and (10), the triangle inequality gives Theorem 3 . 
Function $\tilde{e}$ can be obtained via interpolation techniques, such as radial basis functions, with a goal to minimize $\nu(\tilde{e})$, or alternatively, by solving a simple linear boundary value problem, for example, the Poisson equation, over a coarse mesh for $\Omega-\omega$ with prescribed BCs in Eq. (19). The latter is employed in this paper for ease of implementation with a source term equal to 0 .

\subsection{Discussion}

We now consider several further practical issues: settings for the constants $C_{N}, C_{0}, C_{D}$ discussed earlier, cases with multiple features, and heuristic approaches to estimating modification error.

\subsubsection{Constant settings}

Practical use of the results in Theorems 2 and 3 for estimating modification error still depends on properly setting the constants $C_{N}, C_{0}, C_{D}$. Essentially, no theoretically sound approach exists for finding such constants except in a very few special cases. A previous approach in [28] also utilized similar constants to simplify linear elasticity analysis of perforated materials, but no explicit approach was given for constant setting. In our problem, obtaining exact or tight values of $C_{N}, C_{0}, C_{D}$ is not very essential, since the final modification error estimate also depends on the known terms over the feature's boundary (see Eqs. (14) and (18)), which usually account for a larger contribution to the overall estimated error. We now discuss a practical numerical approach for fining values for these constants.

The derivation of Theorem 2 shows that $C_{N}$ is best set so that Eq. (16) become an equality for solution error $v=e_{0}$, that is,

$$
C_{N}=\frac{\left\|e_{0}\right\|_{L^{2}(\partial \omega)}}{\left\|e_{0}\right\|_{E}}
$$

However, the solution error $e_{0}=u-u_{0}$ is actually unknown. The trace theorem tell us that $C_{N}$ only depends on the geometry of $\Omega-\omega$. Taking into account that Eq. (16) is satisfied for all $v \in V$, we may thus use the space spanned by the computed prime and adjoint solutions $u_{0}, p_{0}$ as a base space, and in practice set $C_{N}$ to be

$$
C_{N}=\max _{s} \frac{\left\|\left(s u_{0} \pm p_{0} / s\right)\right\|_{L^{2}(\partial \omega)}}{\left\|s u_{0} \pm p_{0} / s\right\|_{E}}
$$

This maximal value can be estimated by sampling the parameter $s$ in the range 0 to 1 with say 100 equally spaced steps.

In a similar way, the constants $C_{0}, C_{D}$ may be practically determined using

$$
C_{0}=\max _{s} \frac{\left\|s u_{0} \pm p_{0} / s-\tilde{e}\right\|_{L^{2}(\Omega-\omega)}}{\left\|\operatorname{div}(\nabla \tilde{e})-3 u_{0}^{2} \tilde{e}\right\|_{L^{2}(\Omega-\omega)}},
$$

or simply taken as 1 in $2 \mathrm{D}$ cases, and

$$
C_{D}=\max _{s} \frac{\left\|\nabla\left(s u_{0} \pm p_{0} / s\right)\right\|_{L^{2}(\partial \omega)}}{\left\|\left(s u_{0} \pm p_{0} / s\right)\right\|_{L^{2}(\Omega-\omega)}+\left\|u_{d}-u_{0}\right\|_{L^{2}(\Omega-\omega)}} .
$$

\subsubsection{Multiple features}

The results in Theorems 2 and 3 presume a single feature. We now consider multiple features. Suppose $\omega_{i}, i=$ $1, \ldots, n$, are a set of features to be suppressed within a geometry $\Omega$. Let $\bar{\Omega}=\Omega-\Sigma_{i} \omega_{i}$ be the original geometry, and $\Omega_{i}=\Omega-\omega_{i}$ be the geometry containing a single feature $\omega_{i}$. The results in Theorems $1-3$ do not make any assumption that only a single feature is present, and can thus be applied directly to cases with multiple features. Specifically, the modification error caused by removing all these features $\Omega_{i}$ is simply their summation, that is,

$$
e(\bar{\Omega}, \Omega)=\Sigma_{i} e\left(\Omega_{i}, \Omega\right) .
$$

This allows efficient handling of multiple features as only a single engineering analysis is needed on the fully simplified geometry $\Omega$.

\subsubsection{Other heuristic approaches}

Utilizing the results in Theorems 2 and 3 involves proper estimation of the involved constants, so we also suggest some alternative heuristic approaches based on Theorem 1. While these are not particularly theoretically sound, they do provide alternative, simple, practical approaches.

Consider the two terms in Eq. (10) in Theorem 1, for either Neumann or Dirichlet BC over $\partial \omega$. In either case, one term is known, and the other unknown. Estimates of the unknown terms essentially depend on estimates of $u-u_{0}$ or $\nabla\left(u-u_{0}\right) \cdot n$ along $\partial \omega$.

A heuristic approach to providing them can be based on a local computation strategy, building a local region $\Theta$ surrounding $\omega$, with a corresponding solution $\bar{e}_{0}$ as an approximation to $e_{0}=u-u_{0}$, assuming that $u=u_{0}$ along $\partial \Theta$. Taking into account Eqs. (1) and (3) gives the following equation for $\bar{e}_{0}=u-u_{0}$ :

$$
\begin{cases}L \bar{e}_{0}=0 & \text { in } \Theta-\omega, \\ B \bar{e}_{0}=0 & \text { on } \partial \Theta, \\ D \bar{e}_{0}=d\left(\text { or } u_{d}\right)-D u_{0} & \text { on } \partial \omega,\end{cases}
$$

Computing $\bar{e}_{0}$ only involves a small local region $\Theta$, and is thus much cheaper to compute than computing $e_{0}$ over $\Omega-\omega$. Once a solution $\bar{e}_{0}$ has been found, the modification error can be estimated using Theorem 1. Specifically, for Neumann BCs on $\partial \omega$,

$$
\delta Q \approx-\int_{\partial \omega} \nabla p_{0} \cdot n \bar{e}_{0} d S+\int_{\partial \omega} p_{0}\left(d-d_{0}\right) d S,
$$

while for Dirichlet BCs on $\partial \omega$,

$$
\delta Q \approx-\int_{\partial \omega} \nabla p_{0} \cdot n\left(u_{d}-u_{0}\right) d S+\int_{\partial \omega} p_{0} \nabla \bar{e}_{0} \cdot n d S .
$$

Since the expression in Theorem 10 only depends on the value of $e_{0}$ along $\partial \omega$, the particular choice of $\Theta$ does not greatly change the value of the final estimated errors, and we can thus arbitrarily select $\Theta$ in practice. The solution $\bar{e}_{0}$ can then be computed numerically via a traditional $\mathrm{FE}$ method. A similar approach based on local computation 
was applied in [9] to estimate modification error measured in the global energy norm during linear elasticity analysis. Unlike our approach that only involves the estimated value on the boundary $\partial \omega$, the approach in [9] estimated values over the whole model.

Another simple heuristic strategy to error estimation is to simply discard the unknown term in Eq. (10), resulting in error estimates

$$
\delta Q \approx \int_{\partial \omega} p_{0}\left(d-d_{0}\right) d S
$$

for Neumann BCs, and

$$
\delta Q \approx-\int_{\partial \omega} \nabla p_{0} \cdot n\left(u_{d}-u_{0}\right) d S .
$$

for Dirichlet BCs. The above result for Neumann BCs is consistent with earlier results in [18] based on a different approach; the suggestion for Dirichlet BC is novel.

\subsection{Extension to other nonlinear elliptic problems}

The proposed approach can also be extended to nonlinear second-order elliptic problems. However, concrete expressions for error estimation are very problem-dependent, and need to be derived for each specific case.

Specifically, a nonlinear second-order elliptic problem can be represented in the following form over $\Omega-\omega$ :

$$
L u=\sum_{i} a_{i}(x) \frac{\partial^{2} u}{\partial^{2} x_{i}}+b_{i}(x) \frac{\partial u}{\partial x_{i}}+c_{i}(x), i=2 \text { or } 3
$$

for continuous functions $a_{i}(x), b_{i}(x), c_{i}(x)$.

Following a similar procedure of linear approximation and integration by parts, a result corresponding to Theorem 1 can also be derived for this case without difficulty. This result can be guaranteed for any nonlinear secondorder elliptic problem without requiring any additional properties, as long as the solution exists. Further error estimation, as done in Theorems 2 and 3, are however highly dependent on the particular form of $L u$. For example, even if we simply replaced ' + ' by ' - ' in Eq. (2), solution uniqueness and existence can no longer be guaranteed and the energy norm in Eq. (12) is not properly defined. Correspondingly, the error estimates have to be re-derived based on the result in Theorem 1. The problem of linear elasticity or nonlinear elasticity can be seen as a vector form of Eq. (30) and thus falls into the scope of the above discussion.

\section{Experimental results}

The above approach to estimating modification errors has been implemented using COMSOL [1], a commercial finite-element based CAD/CAE system. We have tested various cases: $2 \mathrm{D}$ internal and boundary features, 3D features, and multiple features with either Neumann or Dirichlet BCs, the features having various sizes and locations.

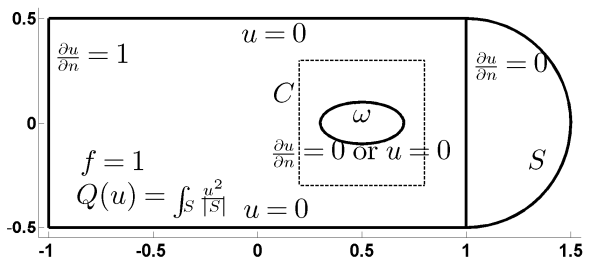

Fig. 3. Example of a 2D internal feature.

The accuracy of an error estimate is usually measured in terms of effectivity index, or EI for short, the ratio between the estimated error $e$ and the ground truth error $E$, that is,

$$
I=e / E
$$

An EI between 0.5 and 2.0 is often taken as reasonable for error measured in a global energy norm. However, for goaloriented error estimation as studied in this paper, obtaining accurate error estimates is generally more challenging, and EIs up to 10 can still be useful in practice [21].

Our experimental results demonstrate performance of three different approaches to error estimation: (i) approximate bounds using Eqs. (14) and (18), denoted Bound in our results; (ii) heuristic-based estimation following Eqs. (26) and (27), denoted Local; (iii) estimation by directly discarding the unknown terms in Eqs. (28) and (29), denoted Direct. All results were compared with ground truth, denoted $Q(u)-Q\left(u_{0}\right)$, obtained via direct FE computations over both the full original geometry and the simplified geometry.

The constants involved in computing Bound were set using the approaches in Section 3.4, while the locally selected regions $\Theta$ involved in computing Local in Eq. (25) were arbitrarily selected around feature $\omega$ and are shown using dashed lines.

\subsection{D internal features}

The simple 2D example in Fig. 3 was first used to test effectivity index values provided by the approach. The analysis problem in Eq. (1) is defined over an original geometry $\Omega-\omega$ for an internal elliptic hole $\omega$ centered at $\left(x_{0}, 0\right)$ with semi-axes of $a$ and $a / 2$; values of $x_{0}, a$ will be described later. Related parameters and BCs are also shown in Fig. 3. A nonlinear local quantity of interest $Q(u)$ is defined over a half disk $S$ on the model's right-hand side as follows:

$$
Q(u)=\frac{\int_{S} u^{2} d S}{|S|}
$$

where $|S|$ stands for the area of $S$.

In this example, error estimates were found for $\omega$ constrained under both homogeneous Neumann BCs and separately, Dirichlet BCs. In each test, the estimated errors and the associated EIs were computed at various sizes and locations of feature $\omega$, with $a$ ranging from 0.01 to 0.1 while $x_{0}=0.5$, or $x_{0}$ ranging from 0.4 to 0.6 while $a=0.1$.

Neumann BCs. We first describe the case when the feature $\omega$ was constrained with Neumann BCs, specifically set 


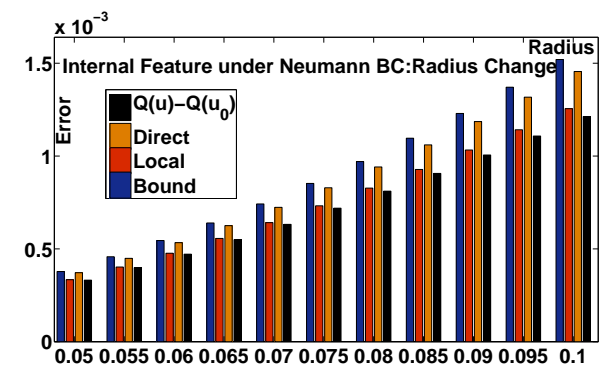

(a) Errors as radius changes

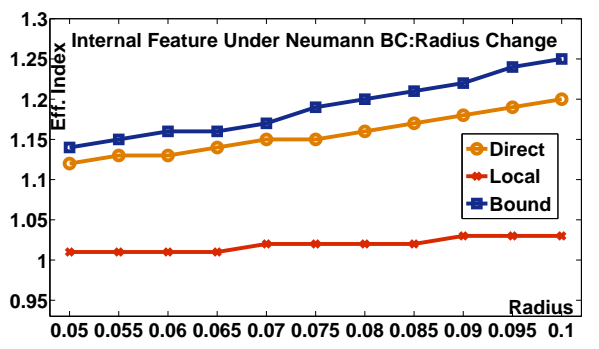

(b) EI as radius changes

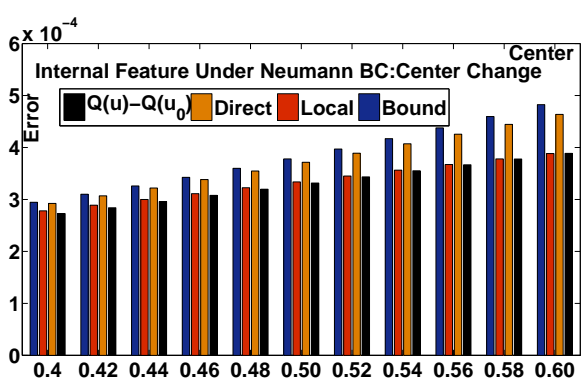

(c) Errors as center changes

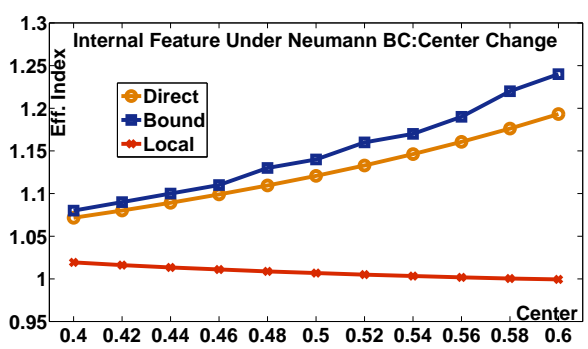

(d) EI as center changes

Fig. 4. Estimated errors and EIs for removal of a varying $2 \mathrm{D}$ internal feature with Neumann BCs, as in Fig. 3.

to $\nabla u \cdot n=0$ on $\partial \omega$. In this case, removing the feature $\omega$ does not cause much error in the target solution $u$ or the quantity of interest $Q(u)$ : the maximal value of $u-u_{0}$ is less than $1 \%$ of the maximal value of $u$.

Estimated errors computed by the three different approaches Bound, Local and Direct, and their associated EIs, are plotted in Fig. 4 and compared with the ground truth. All three different error estimates accurately approximate the ground truth $Q(u)-Q\left(u_{0}\right)$ as the feature's size and location varies. The Direct result, computed by discarding the unknown terms in Eq. (10), has well approximated the ground truth with an EI of about 1.2. Better results were provided by Local, with EIs close to 1.0, due to estimating the unknown term rather than discarding it. The Bound

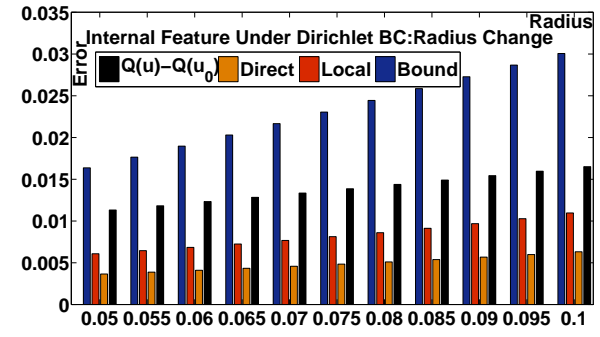

(a) Errors as radius changes

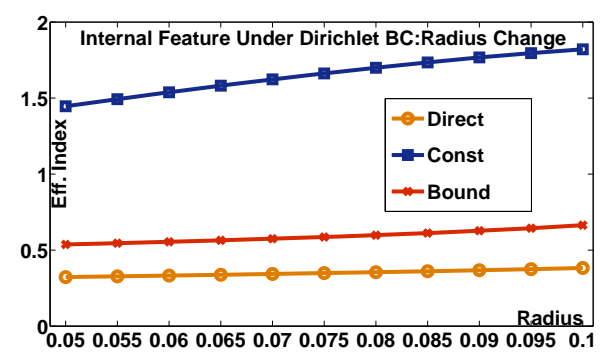

(b) EI as radius changes

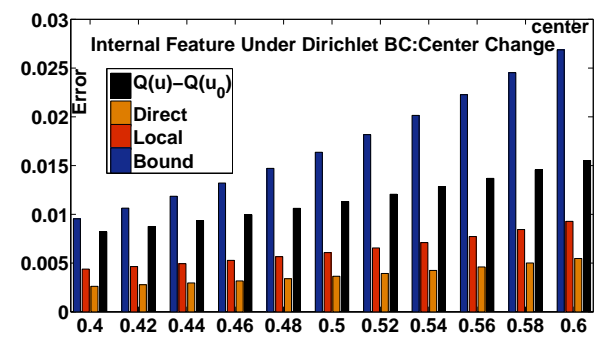

(c) Errors as center changes

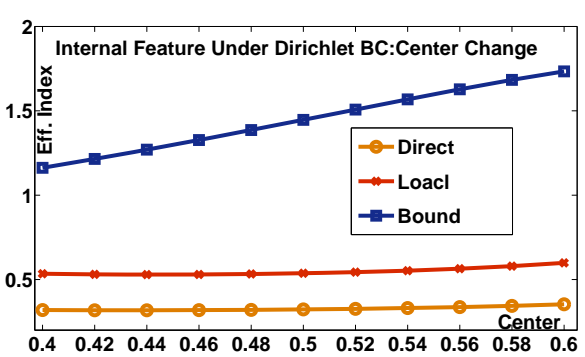

(d) EI as center changes

Fig. 5. Estimated errors and EIs for removal of a varying 2D internal feature with Dirichlet BCs, as in Fig. 3.

result, on the other hand, always bound the ground truth, rather than underestimating it, with EIs ranging from 1.05 to 1.26 .

These results can be explained as follows. Direct approximates the modification error without further estimation of the unknown terms, and thus may underestimate or overestimate the error depending on the positive or negative contribution of the unknown term. Local approximates the unknown term via a local computation, and, as might be expected, generally performs better than Direct; with better effectivity. Bound, on the other hand, aims to give an upper bound on the modification error, and may overestimate the error due to the additional approximation process involved in this bound.

Dirichlet $B C s$. We next consider the case when the fea- 


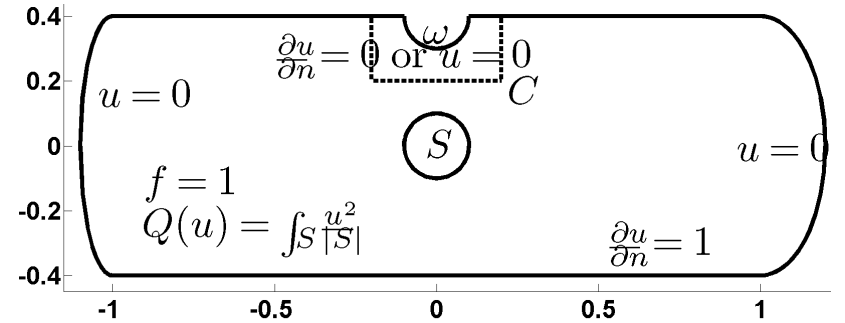

Fig. 6. Example of a 2D boundary feature.

ture $\omega$ was constrained under Dirichlet BCs, set to $u=0$ on $\partial \omega$. Compared to the case with Neumann BCs, removing a feature with Dirichlet BCs makes a much larger change to the solution $u$ and the target quantity $Q(u): u-u_{0}$ is now as large as $u / 3$.

Experimental results are shown in Fig. 4. This time, $D i$ rect had the worse EIs of around 0.3, Local provided EIs from about 0.5 to 0.7 , while Bound provided larger estimates than the others, with EIs ranging from 1.1 to 2.0. In general, the error estimates were worse than those for Neumann BCs. This is mainly due to the much larger error between the solutions $u$ and $u_{0}$, which is assumed to be small in Theorem 1, which hardly holds in this situation. However, the associated EIs still lie in an acceptable range. These results show that the linearization procedure is still useful in practice even for large differences between $u$ and $u_{0}$.

\subsection{D boundary features}

The method can also estimate modification error caused by removing boundary features. This is of practical significance, as most features of industrial CAD parts lie on their boundaries. We first describe experimental results for a $2 \mathrm{D}$ boundary features example shown in Fig. 6, where a semicircular disk $\Omega$, centered at $(0,0.4)$, and with radius $r$ ranging from 0.01 to 0.1 , was cut out from boundary. Further parameters and BCs are described in Fig. (6). A nonlinear local quantity of interest $Q(u)$ was defined over a circular region $S$ in the same form as in Eq. (31).

Experimental results for Bound, Local and Direct are plotted in Fig. 7. Both Direct and Local provide reasonable error estimates with the worse EI around 0.5. Unlike the experimental results in Section 4.1, Local does not always perform better than Direct. For example, in the case of Neumann BC shown in Fig. 7(b), Local and Direct provide approximately the same results for $r$ ranging from 0.01 to 0.04 . However, as $r$ ranged from 0.04 to 0.1 , Direct performed slightly better than Local, with the worse EI respectively 0.988 and 0.976 .

The Bound estimate always bounds the ground truth except for small radius features with Neumann BC when the radius is small (see Fig. $7(\mathrm{~b})$ ), whereupon the EI drops to slightly smaller than 1.0. This may be due to the linearization process in Theorem 1, causing approximation errors. The estimated modification errors are very small values,

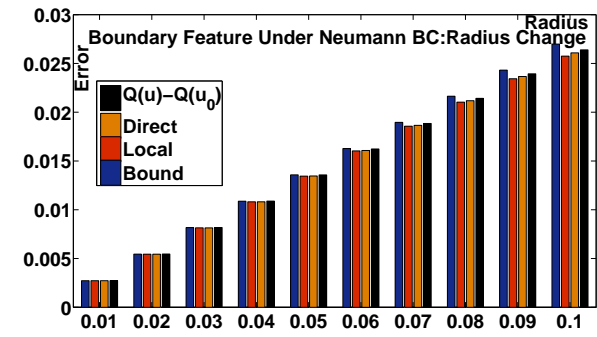

(a) Errors for Neumann BC

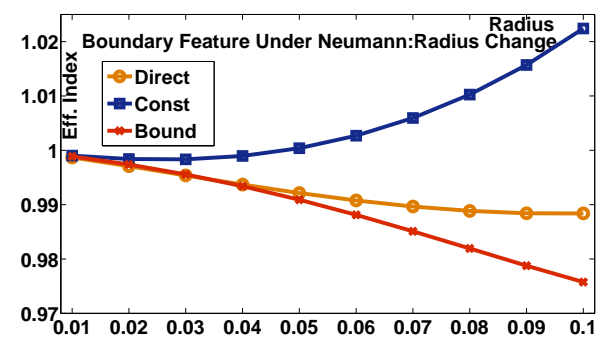

(b) EI for Neumann BC

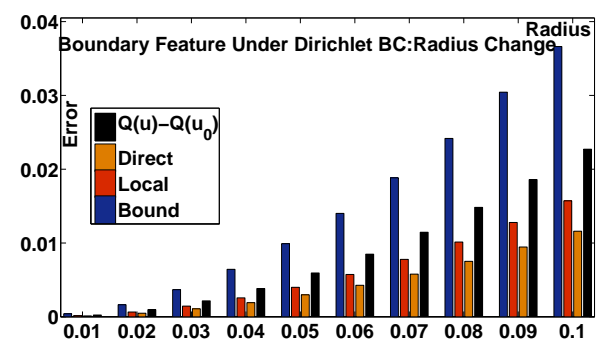

(c) Errors for Dirichlet BC

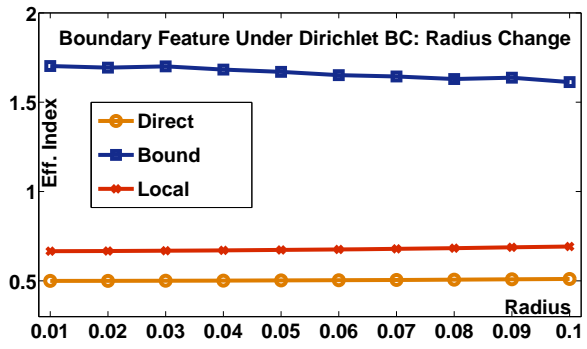

(d) EI for Dirichlet BC

Fig. 7. Estimated errors and EIs for changing feature size, when removing a 2D boundary feature with Neumann or Dirichlet BCs, as in Fig. 6.

and may also be subject to numerical errors. The error estimates generally perform better in the case of Neumann BC than for Dirichlet BCs, with EIs respectively between 0.97 to 1.03 , and 0.5 to 1.8 .

\subsection{D features}

The simple 3D example in Fig. 8 was also tested. A cylindrical hole $\omega$ was cut out of a cylindrical base. The source term $f$ in Eq. (1) was set to $f=1$, the base face was constrained by $u=0$, the top face was constrained by $\nabla u \cdot n=$ 1 , and the side face was set free. The internal feature was constrained using either Neumann or Dirchlet BCs, each with a different local quantity of interest $Q(u)$ defined over 


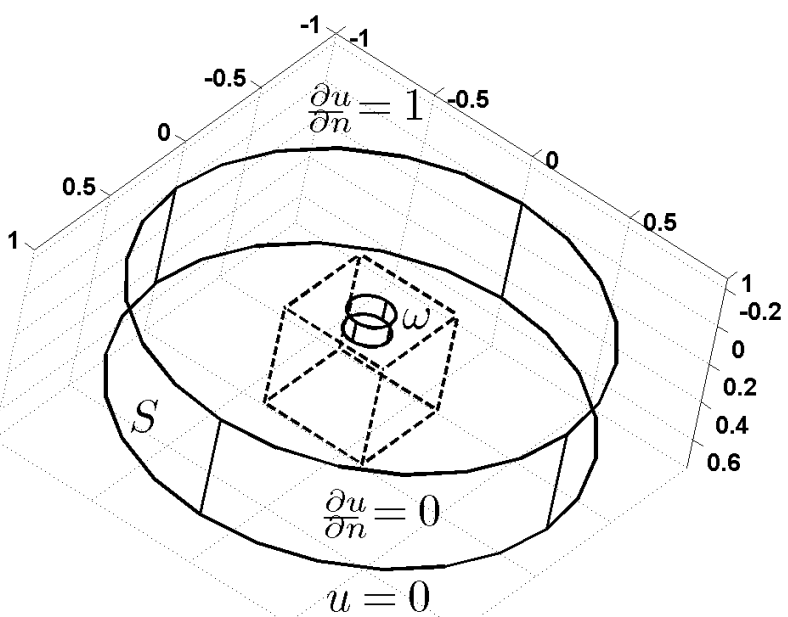

Fig. 8. Example of a 3D feature.

the side face $S$ as follows

$$
Q(u)=\frac{\int_{S} u_{z} d S}{|S|} \text { or } \quad Q(u)=\frac{\int_{S} u d S}{|S|} .
$$

Experimental results are plotted in Fig. 9. Figs. 9(a),(b) show the results when the internal feature had homogeneous Neumann BC and the cylindrical hole's radius varied from 0.02 to 0.2 . All error estimaties are close to the ground truth, with EIs ranging from 0.98 to 1.13. Using additional local computations, Local performed better than Direct, and Bound tightly bounds the ground truth with EIs ranging from 1.0 to 1.13. Direct and Bound produced almost the same estimated errors.

Figs. 9(c),(d) show the obtained results when the internal feature was constrained using homogeneous Dirichelt BC and the cylindrical hole's height is varied from 0.05 to 0.5 . Note that when the height is equal to 0.5 , the cylindrical blind hole becomes a through hole. In this example, Direct was rather far from the ground truth with EIs around 0.25. Local performed better with EIs around 0.6. Bound bounds the ground truth with EIs ranging from 1.4 to 2.3.

\subsection{D multiple features}

We also tested the performance of the proposed error estimates for the case of multiple features on an industrial component $\bar{\Omega}$ in Figure 10 containing twelve features denoted $F_{1}, \ldots, F_{12}$. Removing all $F_{i}$ from $\bar{\Omega}$ results in a fully simplified geometry $\Omega$. In this example, the right hand end was constrained at $u=0$. Other boundaries were set free. $\mathrm{BCs}$ for each feature were set as follows:

$$
\begin{aligned}
& F_{1}: \text { free; } F_{2}: u=0 ; F_{3}: \text { free; } F_{4}: u=0 \\
& F_{5}: \text { free; } F_{6}: u=0 ; F_{7}: \text { free; } F_{8}: \nabla u \cdot n=10 \\
& F_{9}: \text { free; } F_{10}: \text { free; } F_{11}: \text { free; } F_{12}: \text { free }
\end{aligned}
$$

The corresponding solution $u$ of the original geometry $\bar{\Omega}$, and solution $u_{0}$ of the fully simplified geometry $\Omega$ are plot-

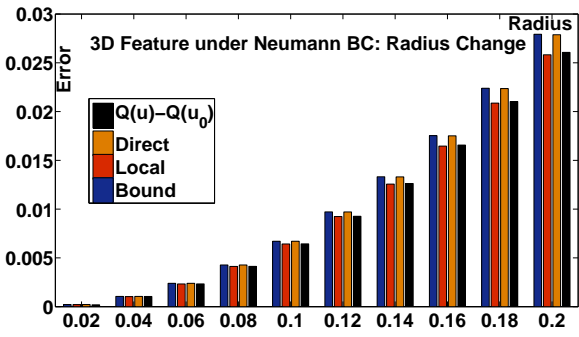

(a) Errors as radius changes for Neumann BC

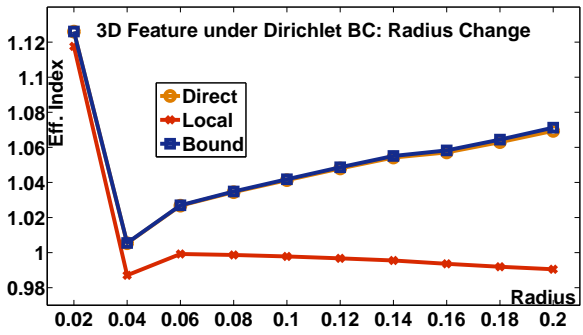

(b) EI as radius changes for Neumann BC

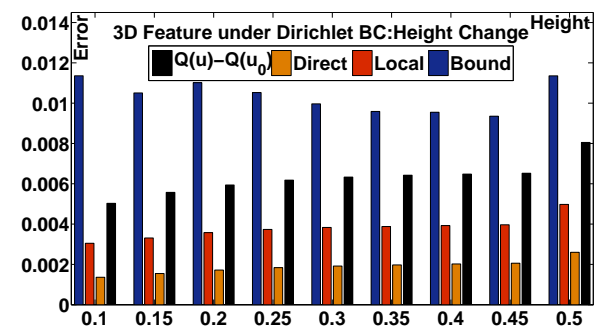

(c) Errors as height changes for Dirichlet BC

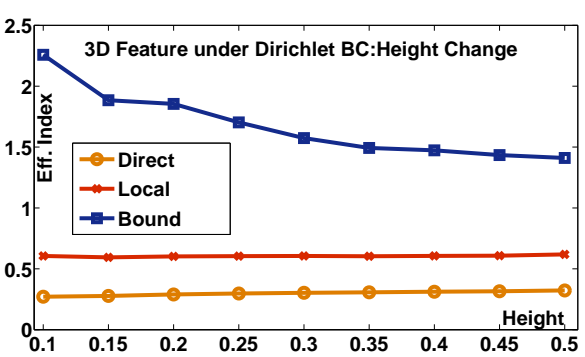

(d) EI as height changes for Dirichlet BC

Fig. 9. Estimated errors and EIs for removing a 3D boundary feature with vary radius or height, with Neumann or Dirichlet BCs, for the example in Fig. 8.

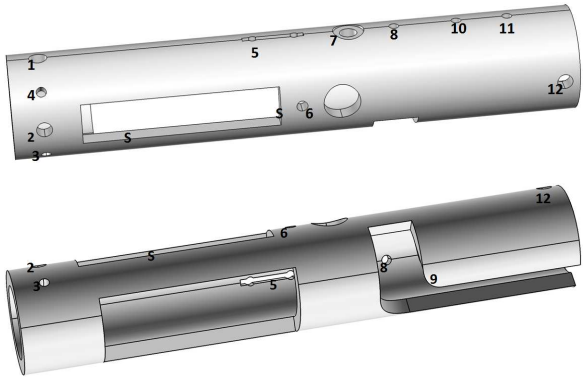

Fig. 10. An engineering component containing various features.

ted in Figure 11(a),(b). Big differences between the two solutions can be clearly seen in this example: the solution for the original model ranged between $0.0-0.5$, while the solu- 


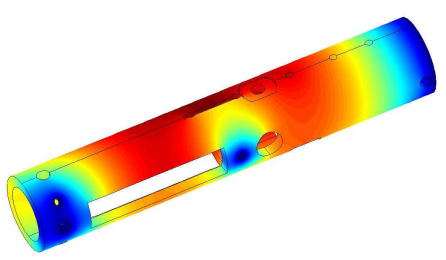

(a) Solution on original model
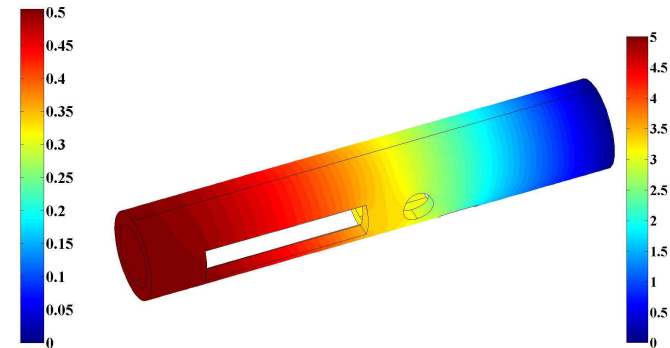

(b) Solution on fully simplified model

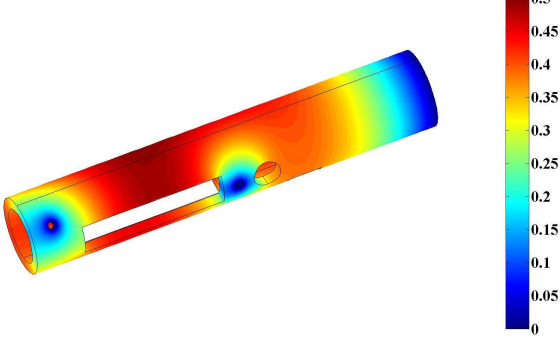

(c) Solution on partially simplified model

Fig. 11. Engineering analysis solutions for the original model, the partially simplified model, and the fully simplified model.

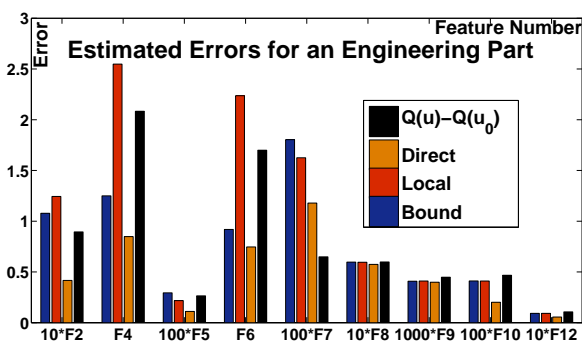

(a) Errors

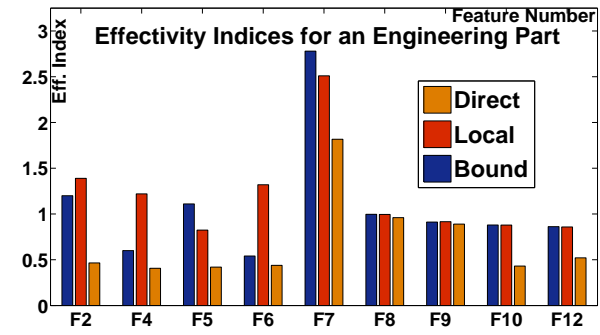

(b) EIs

Fig. 12. Estimated errors and associated EIs for removing a single 3D feature with either Neumann or Dirichlet BCs for the example in Fig. 10.

tion for the fully simplified model ranged between 0.0-5.0.

A local quantity of interest was defined over the boundaries of a four-sided hole $S$ (see Fig. 10) as follows:

$$
Q(u)=\frac{\int_{S} u d S}{|S|},
$$

where $|S|$ stands for the area of $S$.

The modification error for removing a feature $F_{i}$ was estimated as $e\left(\Omega_{i}, \Omega\right)$. Taking the simplified model as $\Omega$ is very important for computational efficiency when there are multiple features: the simplified model remains the same for all features, so error estimation only involves one-time engineering analysis over $\Omega$.

The estimated errors and EIs for each feature are plotted in Fig. 12; features $F_{1}, F_{3}, F_{11}$ are all ignorable and thus not further shown. As can be seen from the results, ranges of the EIs are $0.4-2.0$ for Direct, $0.8-2.6$ for Local. Ranges of the EIs for Bound lie between 0.55 to 3.0, and do not necessarily bound the ground truth. This situation can perhaps be explained in two ways. Firstly, the result in Theorem 1 was obtained via a linear approximation process by assuming the difference between $u$ and $u_{0}$ is small. However, some
Table 1

Comparisons of the computational results between the original model $\bar{\Omega}$, the fully-defeatured model $\Omega$, and the partially defeatured model $\Omega_{m}$ obtained by the proposed error estimation approaches.

\begin{tabular}{|l|r|r|r|r|}
\hline Model & Quantity & Error & Mesh elements & Running time \\
\hline $\bar{\Omega}$ & 0.317 & 0 & 58744 & $7.8 \mathrm{~s}$ \\
\hline$\Omega$ & 2.891 & 2.574 & 28784 & $4.0 \mathrm{~s}$ \\
\hline$\Omega_{m}$ & 0.379 & 0.062 & 32036 & $5.4 \mathrm{~s}$ \\
\hline
\end{tabular}

features, for example $F_{4}$, are key to the final analysis results and do not satisfy this assumption, as can be observed from the error estimates in Fig. 12 and the plotted solutions in Fig. 10. Secondly, the value of the constants $C_{N}, C_{0}, C_{D}$ are only estimated via the approaches in Section 3.4.1, and may be under-estimates.

Different features have different importance to the target quantity $Q(u)$. In particular, features $F_{4}$ and $F_{6}$ are much more important than other features, and should thus be retained within the final simplified model while other unimportant features may be removed. This results in a partially simplified model, whose corresponding analysis solution is shown in Fig. 11(c). This solution is now visually similar to that in Fig. 11(a).

Comparisons between analysis of the three different models, the original model $\bar{\Omega}$, the fully simplified model $\Omega$, and the partially simplified model $\Omega_{m}$ are also shown in Table 1 . As can be seen from these results, using the fully defeatured model $\Omega$ saved some computational time but produced large analysis errors. Using the partially simplified model $\Omega_{m}$ again reduced the analysis complexity and the computational time, while simultaneously maintaining high analysis accuracy. Such performance would be very difficult to achieve simply using geometry-based simplification techniques.

\section{Conclusions and future work}

Three different approaches for estimating modification error have been suggested in this paper for estimating goaloriented modification error induced by removing negative features from a complex model. The features may lie within the model's interior or along its boundary, and may be constrained with either Neumann or Dirichlet BCs. (The result of Direct on Neumann BC is identical with a previous 
result in [18], but was obtained using a different approach.)

Our experimental results for both 2D and 3D internal boundary features show that all three error estimates approximate the ground truth reasonably well for a feature with either Neumann or Dirichlet BC. Comparing the three different approaches, Direct involves heuristics and is simplest, and generally demonstrates lower effectivity than the other two. Local is very stable and also involves heuristics; it improves Direct via an additional local computation for each candidate feature. Error Bound is mathematically rigorous, and builds approximate bounds for the estimated errors, but needs estimation of certain constants. These three different results may serve for different application purposes, or be used together to boost confidence in the estimated errors. The estimated errors can be used to guide geometry simplification while maintaining engineering analysis fidelity.

Future work is now discussed. Firstly, the result in Theorem 1 only holds approximately due to linearization. The resulting linearization error should be further considered in order to build a final estimate that strictly bounds the modification errors.

Secondly, the constants in Theorems 2 and 3 must be accurately determined in order for the estimated error Bound to be close to the ground truth. They are determined by maximizing an expression in the space expanded by the computed solutions $u_{0}, p_{0}$, which improves the robustness over simply using $u_{0}$ or $p_{0}$. However, better approaches for setting these constants than sampling need to be explored, to avoid unexpected poor choices for these constants.

Thirdly, Local error is estimated via an additional local computation for each feature, which provides better EIs, as experimentally demonstrated. On the other hand, it would be an improvement to avoid heuristics. Theoretically rigorous approaches need to be further developed to optimally select the region used, or to explore the effect of region selection on the final estimated errors. Furthermore, by appropriately setting the values of $u$ along the boundary $\Theta$, we may build an error approximation with verified convergence, or even strict lower and upper bounds on the estimated errors. Extending the approach in [15] may achieve this. Note that the proposed approach of local region selection is different from multigrid methods or domain decomposition methods in that the former aim to properly estimate the modification error, which may be small or large, while the latter aim to find the target exact solution, ignoring numerical approximation error, via local iterative computation strategies.

Fourthly, the approach relies on the original model being contained within the simplified model, which does not hold in the case of positive features. A heuristic approach via local solution extension has been proposed in [17] to resolve this issue, which can also be applied here for cases of positive features. However, this approach still lacks a rigorous theoretical verification, and deserves further research effort.

Lastly, applying the proposed approach to generating a simplified geometric model with engineering analysis error control is a non-trivial task and needs to be further explored. More work is needed on estimation of the engineering analysis error caused by removing multiple features, which may interact, and on optimal selection of features for removal. Furthermore, geometric approaches are needed to automatically detect candidate features to be suppressed, and to suppress them, in cases both where feature information is part of the model, and where it must be deduced from the geometry alone. An approach towards this goal can be found in [22].

\section{Acknowledgements}

The work described in this paper was partially supported by the National Basic Research Program of China (No. 2011CB302400), the NSF of China (No. 61103103). We thank Dr. Kai Zhang for his helpful discussions on the manuscript, and Junzhe Zheng for implementing the tests.

\section{References}

[1] COMSOL multiphysics 3.5a. http://www.comsol.com., 2007.

[2] S. Amstutz. Sensitivity analysis with respect to a local perturbation of the material property. Asymptotic Analysis, 49(1-2):87-108, 2006.

[3] M. Badiale and E. Serra. Semilinear Elliptic Equations for Beginners. Springer, 2011.

[4] Y. Bazilevs, V. Calo, J. Cottrell, J. Evans, T. Hughes, S. Lipton, M. Scott, and T. Sederberg. Isogeometric analysis using T-splines. Computer Methods in Applied Mechanics and Engineering, 199(5-8):229-263, 2010.

[5] R. Becker and R. Rannacher. An optimal control approach to a posteriori error estimation in finite element methods. Acta Numerica, 10:1-102, 2001.

[6] G. Caloz and J. Rappaz. Handbook of numerical analysis, chapter Numerical analysis for nonlinear and bifurcation problems, pages 487-638. 1997.

[7] K. Choi and N. Kim. Structural Sensitivity Analysis and Optimization I: Linear Systems. Springer, New York, 2005.

[8] L. Evans. Partial Differential Equations. American Mathematical Society, New York, 1998.

[9] R. Ferrandes, P. Marin, J. Leon, and F. Giannini. A posteriori evaluation of simplification details for finite element model preparation. Computers \& Structures, 87(1-2):73-80, 2009.

[10] M. Giles and E. Suli. Adjoint methods for PDEs: a posteriori error analysis and postprocessing by duality. Acta Numerica, 11:145-236, 2002.

[11] S. Gopalakrishnan and K. Suresh. Estimating the impact of large design changes on field problems. In ACM Symposium on Solid and physical modeling, pages 205-215, 2007.

[12] S. Gopalakrishnan and K. Suresh. A formal theory for estimating defeaturing-induced engineering analysis errors. ComputerAided Design, 39(1):60-68, 2007.

[13] T. Gratsch and K. Bathe. A posteriori error estimation techniques in practical finite element analysis. Computers \& Structures, 83(4-5):235-265, 2005.

[14] T. Hughes, J. Cottrell, and Y. Bazilevs. Isogeometric analysis: CAD, finite elements, NURBS, exact geometry and mesh refinement. Computer Methods in Applied Mechanics and Engineering, 194(39-41):4135-4195, 2005.

[15] J. Keller. Removing small features from computational domains. Journal of Computatinal Physics, 113(1):148-150, 1994. 
[16] D. Kelly, J. De, S. Gago, O. Zienkiewicz, and I. Babuska. A posteriori error analysis and adaptive processes in the finite element method: Part I: error analysis. International Journal for Numerical Methods in Engineering, 19(11):1593-1619, 1983.

[17] M. Li and S. Gao. Estimating defeaturing-induced engineering analysis error for arbitrary 3D features. Computer-Aided Design, 43(12):1587-1597, 2011.

[18] M. Li, S. Gao, and R. Martin. Estimating effects of removing negative features on engineering analysis. Computer-Aided Design, 43(1):1402-1212, 2011.

[19] J. Oden and S. Prudhomme. Estimation of modeling error in computational mechanics. Journal of Computational Physics, 182(2):496-515, 2002.

[20] S. Owen. A survey of unstructured mesh generation technology. In 7th International Meshing Roundtable, pages 239-267, 1998.

[21] S. Prudhomme, J. Oden, T. Westermann, J. Bass, and M. Botkin. Practical methods for a posteriori error estimation in engineering applications. International Journal for Numerical Methods in Engineering, 56(8):1193-1224, 2003.

[22] W. Quadros and S. Owen. Defeaturing CAD models using a geometry-based size field and facet-based reduction operators. In Proceedings of the 18th International Meshing Roundtable, pages 301-328, 2009.

[23] B. Russ, M. Dabbeeru, A. Chorney, D. Skelley, and S. Gupta. Suppressing features to generate simplified models for finite element analysis. In ASME IDETC/CIE, 2011.

[24] V. Shapiro, I. Tsukanov, and A. Grishin. Geometric issues in computer aided design/computer aided engineering integration. Journal of Computing and Information Science in Engineering, 11(2):021005-1:13, 2011.

[25] J. Sokolowski and A. Zochowski. On topological derivative in shape optimization. SIAM Journal of Control Optimization, 37(4):1251-1272, 1999.

[26] A. Thakur, A. Banerjee, and S. Gupta. A survey of CAD model simplification techniques for physics-based simulation applications. Computer-Aided Design, 41(2):65-80, 2009.

[27] I. Turevsky, S. Gopalakrishnan, and K. Suresh. Defeaturing: A posteriori error analysis via feature sensitivity. International Journal for Numerical Methods in Engineering, 76(9):1379-1401, 2008.

[28] K. Vemaganti. Modelling error estimation and adaptive modelling of perforated materials. International Journal for Numerical Methods in Engineering, 59(12):1587-1604, 2004. 